\title{
GridSample: an R package to generate household survey primary sampling units (PSUs) from gridded population data
}

Dana R. Thomson 1,2,3* Forrest R. Stevens ${ }^{3,4}$, Nick W. Ruktanonchai ${ }^{2,3}$, Andrew J. Tatem ${ }^{2,3}$ and Marcia C. Castro ${ }^{5}$

\begin{abstract}
Background: Household survey data are collected by governments, international organizations, and companies to prioritize policies and allocate billions of dollars. Surveys are typically selected from recent census data; however, census data are often outdated or inaccurate. This paper describes how gridded population data might instead be used as a sample frame, and introduces the R GridSample algorithm for selecting primary sampling units (PSU) for complex household surveys with gridded population data. With a gridded population dataset and geographic boundary of the study area, GridSample allows a two-step process to sample "seed" cells with probability proportionate to estimated population size, then "grows" PSUs until a minimum population is achieved in each PSU. The algorithm permits stratification and oversampling of urban or rural areas. The approximately uniform size and shape of grid cells allows for spatial oversampling, not possible in typical surveys, possibly improving small area estimates with survey results.

Results: We replicated the 2010 Rwanda Demographic and Health Survey (DHS) in GridSample by sampling the WorldPop 2010 UN-adjusted $100 \mathrm{~m} \times 100 \mathrm{~m}$ gridded population dataset, stratifying by Rwanda's 30 districts, and oversampling in urban areas. The 2010 Rwanda DHS had 79 urban PSUs, 413 rural PSUs, with an average PSU population of 610 people. An equivalent sample in GridSample had 75 urban PSUs, 405 rural PSUs, and a median PSU population of 612 people. The number of PSUs differed because DHS added urban PSUs from specific districts while GridSample reallocated rural-to-urban PSUs across all districts.

Conclusions: Gridded population sampling is a promising alternative to typical census-based sampling when census data are moderately outdated or inaccurate. Four approaches to implementation have been tried: (1) using gridded PSU boundaries produced by GridSample, (2) manually segmenting gridded PSU using satellite imagery, (3) nonprobability sampling (e.g. random-walk, "spin-the-pen"), and random sampling of households. Gridded population sampling is in its infancy, and further research is needed to assess the accuracy and feasibility of gridded population sampling. The GridSample R algorithm can be used to forward this research agenda.
\end{abstract}

Keywords: Cluster survey, Multi-stage, Cluster sample

\section{Background}

Household survey data are collected to support prioritization of national and international issues, allocate billions of donor and government dollars, track progress toward major policy and program goals including the sustainable development goals (SDGs) [1, 2], quantify

\footnotetext{
*Correspondence: dana.r.thomson@gmail.com

1 Department of Social Statistics and Demography, University

of Southampton, Building 58, Southampton SO17 1BJ, UK

Full list of author information is available at the end of the article
}

needs during disaster responses [3, 4], and follow consumer trends [5]. Household surveys are particularly important in countries where census data, or other forms of official data such as birth and death registries, are outdated, incomplete or inaccurate. Selection of representative household survey samples requires definition of areal units with up-to-date and accurate population counts-typically enumeration areas from a recent census-creating a circular dilemma. Where census data are not available, outdated, or known to be unreliable, 
individual survey teams have begun to experiment with gridded population sampling as an alternative [6-11], and organizations that fund routine surveys are beginning to recommend gridded population datasets as alternative sample frames [12]. To date, however, no tools exist to support complex survey selection from gridded population datasets, and there is scant guidance to use these emerging methods. This paper (1) describes how gridded population datasets have been used as alternative sample frames to outdated or inaccurate census data, (2) introduces GridSample [13], an R package, for the first-stage selection of complex household surveys using gridded population data, and (3) summarizes options to implement gridded population samples in the field. $R$ is an open-source free software environment created and maintained by hundreds of developers from many disciplines worldwide. $\mathrm{R}$ contains well-established, usercreated packages to perform statistical analysis and data visualization.

\section{Typical household surveys}

Since the 1980s, hundreds of nationally-representative household surveys have been conducted by governments in low- and middle-income countries roughly every five years with support from the United Nations (UN) [14, 15], the US Government [16], and the World Bank [17] to monitor social, demographic, economic, and health indicators. The UN's Multiple Indicator Cluster Surveys
(MICS), the US Government's Demographic and Health Surveys (DHS), and the World Bank's Living Standard Measurement Surveys (LSMS) stratify samples by sub-national region, and sample roughly 10,000 households in a two-stage design that is widely used by survey implementers to maximize statistical power and feasibility while minimizing costs and potential biases [14-16]. Each of these surveys cost several hundred thousand US dollars and approximately two years to implement and publish [18].

In standard large-scale household surveys, implementers sample communities first (called clusters, or primary sampling units-PSUs) from recent census enumeration areas. Then second, list all households in the sampled communities during a field mapping exercise before systematically sampling households [13, 15, 16] (Fig. 1). In the poorest settings, household enumeration is still routinely performed by hand with a pencil and paper [16], and satellite-enhanced enumeration has been piloted with printed maps of satellite imagery and with mobile devices $[19,20]$. While these methods are widely adopted and considered the gold-standard, they are limited in their ability to generate accurate samples when census data frames are outdated or inaccurate [21]. At the time of this writing in 2017, 37 of 157 countries in Africa, Asia, and Central and South America has a census that is 10 years old or more [22]. Many of these countries have experienced population displacement by environmental

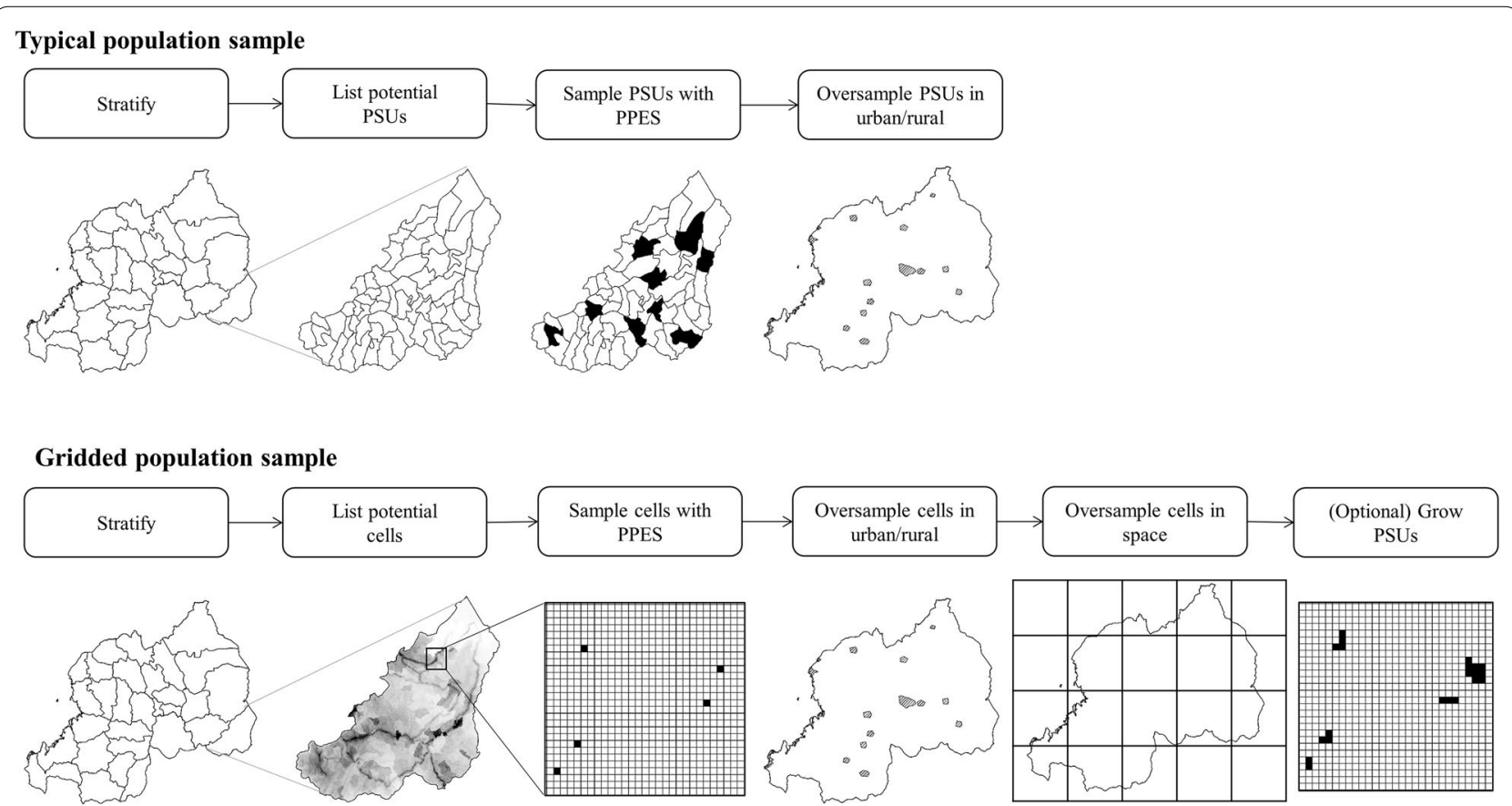

Fig. 1 Comparison of first stage in typical population sampling and gridded population sampling 
disasters, conflict, rapid economic change [23], official changes to subnational administrative area boundaries [24] and normal demographic shifts due to changing birth and death rates.

\section{Gridded population data}

Gridded population data may prove to be a viable alternative sample frame where census data are outdated or inaccurate. Three types of gridded population datasets are available. First, standard "top-down" gridded population datasets are generated by models that either directly disaggregate administrative population counts to grid cells using satellite imagery (e.g. land cover and nighttime lights) and other spatial data (e.g. road and building locations), or non-uniformly disaggregate population counts using complex modeling approaches. Direct disaggregation approaches vary from simple areal weighting (e.g. GPWv4 [25], UNEP [26]) to use of ancillary data, such as urban settlement areas, to inform the location and density of disaggregated population (e.g. GRUMP [27], GHSPop [28], Facebook [29]). Complex modelling techniques (e.g. WorldPop [30], Landscan [31], Demobase [32]) include such methods as aggregating input and covariate data at two scales to test and tailor the model to local areas.

Multiple top-down global gridded population datasets are available to freely download including WorldPop [33], GPWv4 [34], GHS-POP [35], GRUMP [36], and UNEP [26]. Landscan [37] datasets are free to US Federal Government agencies and some humanitarian, education and commercial organizations, upon request. Gridded population datasets are published as population estimates per pixel, where pixels are measured in decimal degrees and are thus slightly smaller and less square-shaped toward the earth's poles compared to the equator. Within countries, differences in cell size are generally negligible; exceptions include Brazil and Russia with large northsouth coverage. WorldPop [33] additionally provides population per hectare estimates measured in meters, where each pixel is $100 \mathrm{~m} \times 100 \mathrm{~m}$ anywhere on earth. Gridded population datasets have known inaccuracies, particularly at the sub-national and metropolitan scales $[38,39]$. Although top-down gridded population datasets may be based on outdated or incorrect population totals from 2nd-, 3rd-, and 4th-level administrative areas, the distribution of population estimates within administrative areas might be more representative of the population than enumeration area counts in the last census.

Gridded population data need not be based entirely on census data. Where census data are grossly outdated and populations are reasonably stationary, researchers are experimenting with a second type of gridded population dataset using "bottom-up" methods that integrate population counts from small area surveys with dozens of spatial covariates [40]. In areas where large-scale population movement has resulted from a major event, such as an earthquake or violent conflict, researchers have begun to work with mobile phone companies to gain anonymized, aggregated call detail records (CDR) and generate a third type of CDR-enhanced gridded population dataset [41-43].

\section{Gridded population sampling for household surveys}

The GridSample package was recently released in $\mathrm{R}$ CRAN to generate PSUs for household surveys using gridded population data rather than census data [13]. GridSample supports typical complex sample designs including stratification, oversampling in urban or rural areas, and sampling of different numbers of households within urban and rural areas (Fig. 1). Because grid cells are approximately uniform in size and shape within a country, GridSample also allows for a population sample to be supplemented with a spatial oversample in remote areas which is attractive if survey results will be used to generate small area estimates or make interpolated surfaces [44] (Fig. 1).

The user needs either two or three datasets to use GridSample. First, a gridded population dataset that covers the study area. Gridded population data are produced in raster file format. A common example of a raster dataset is a photograph which is comprised of pixels, each with a single color value. Similar to a photograph, gridded population cells each have one estimated population value. Second, the user provides the boundary of the study area if the sample is not stratified, or boundaries of geographic strata if the sample is stratified. Third, the user optionally inputs urban/rural area boundaries if urban and rural domains will be represented in the survey. Boundaries are commonly formatted as a shapefile, a type of file used to store points, lines, or polygons (areas) and their attributes. GridSample requires that all input datasets are converted to raster format using the same grid cell dimensions as the population dataset. Below, we provide a code example to convert shapefiles to rasters.

The input raster datasets, plus a number of survey parameters, are used to randomly sample grid cells with probability proportionate to estimated population (PPES) size in a first step, and then optionally grow PSUs around selected seed cells until a minimum population threshold is achieved in a second step (Fig. 2). This two-step process ensures both that the desired number of PSUs per strata and domain are achieved, as well as desired population per PSU. GridSample outputs a shapefile of PSU boundaries which can be visualized in a geographic information system like $\mathrm{QGIS}^{\mathrm{TM}}$ or $\mathrm{ArcGIS}^{\mathrm{TM}}$, or overlaid on satellite imagery, for example in Google Earth ${ }^{\mathrm{TM}}$. 
The shapefile includes a record for each PSU containing the latitude-longitude coordinate of the PSU centroid (geographic center), and the PSU and strata population counts needed in sample weight calculations.

In the following sections, we provide a technical overview of the GridSample algorithm workflow; describe how to replicate typical complex survey designs in GridSample; describe the use of population sampling with a spatial oversample; and reproduce an existing DHS sample in GridSample. To support use of GridSample, we provide sample weight calculation instructions, discuss practical limitations, outline areas for future gridded population survey research, and offer suggestions to improve the feasibility of fieldwork.

\section{GridSample: technical workflow}

GridSample is an R CRAN package with four functionsgs_mode, gs_rasterize, gs_zonal_raster, and gs_samplethough the user only interacts with the main function, gs_sample. GridSample is written for $\mathrm{R}$ version 3.2 .3 or newer, and requires the following libraries: rgdal $(\geq 1.2-$ $5)$, raster $(\geq 2.5-8)$, data.table $(\geq 1.10 .4)$, rgeos $(\geq 0.3-22)$, geosphere ( $\geq 1.5-5)$, sp $(\geq 1.2-4)$, deldir $(\geq 0.1-12)$, spatstat $(\geq 1.49-0)$, and maptools $(\geq 0.8-41)$. Figure 2 visualizes how the input datasets and parameters are processed in gs_sample. At a minimum, the user must specify the input gridded population dataset (population_raster), household sample size (cfg_hh_per_stratum), study area boundary (which is strata raster, the boundary of a single stratum sample), population size per PSU (cfg pop per psu), and number of households to be sampled per PSU (the urban value $\mathrm{cfg}$ hh per urban is used for all PSUs if a rural value $\mathrm{cfg}_{\mathrm{g}} \mathrm{hh}$ per rural is not specified). Further complexities can be added to the survey design including stratification, oversampling of urban/rural populations, and spatial sampling. GridSample first selects PSU seed cells from the dataset, and then optionally grows each PSU by adding neighboring cells until a minimum geographic size (cfg_max_psu_size) or population size (cfg_pop per_psu) is achieved.

Before using gs_sample, the user must rasterize all vector data to match the grid dimensions of the gridded population dataset (population_raster). Specifically, the user must rasterize urban/rural boundaries and strata boundaries. Urban/rural boundaries (urban raster) may be defined from existing data sources such as Global Urban Footprint (GUF) [45], Global Rural Urban Mapping Project (GRUMP) [36], Global Human Settlement City Model (GHS-SMOD) [46], Modis $500 \mathrm{~m}$ urban extents [47], and European Space Agency Land Cover class for urban areas [48]. Alternatively, the user may generate urban/rural extents by classifying the population density layer (population raster), or by uploading an urban/rural shapefile from another source. Choice of urban/rural boundary is highly dependent on the nature of the survey, as definitions of urban and rural populations differ across countries and disciplines [49]. The strata boundary raster (strata_raster) can be derived from administrative area boundaries, for example Map Library [50] or DIVA-GIS [51], though the user might upload alternative strata boundaries defining, for example, ecological regions or a program catchment area.

To select PSU seed cells, gs_sample classifies each cell in the gridded population dataset (population_raster) by urban or rural location (if $\mathrm{cfg}$ sample rururb = TRUE and urban raster is specified), and assigns a stratum ID (strata raster). Serpentine sampling is used such that cells are geographically ordered from west-to-east, north-tosouth, and sampled based on a random starting cell and a population increment that produces the desired number of PSUs within the stratum, thus facilitating a randomized population-weighted sample. The user may halt the algorithm at this point leaving just one cell per PSU by setting the PSU growth parameter to false (cfg_psu_growth = FALSE).

If the PSU growth parameter is set to true (cfg_psu_ growth $=$ TRUE), gs_sample grows PSUs using a dilation filter routine to enlarge the area around each PSU seed cell by adding neighboring cells one cell at a time until the specified population per PSU parameter is met. From the seed cell, the dilation routine randomly chooses one of the nearest north, east, south, or west cells, and adds that population to the PSU. The routine loops over each PSU adding more population cells each time until each PSU achieves the maximum PSU area in square kilometers (cfg_max_psu_size) or total population per PSU value (cfg_pop_per_psu). A valid sample frame has contiguous, non-overlapping potential PSUs. Thus, GridSample restricts PSUs to being contiguous and nonoverlapping by drawing voronoi polygons around each seed cell, defining unique areas in which each PSU can grow; the PSU growth routine will not add cells beyond a strata or voronoi polygon boundary.

After all PSUs have been selected, the algorithm generates a polygon shapefile of the PSU boundaries and assigns the following attributes to each PSU: PSU identifier, stratum identifier, urban/rural class of the seed cell, PSU centroid coordinate, total/urban/rural population in PSU, total/urban/rural population in stratum, number of cells in PSU, and number of PSUs in stratum (Table 1). The algorithm prints to screen the value of the random number used to start the sampling process; this value can be recorded and manually entered in GridSample to 
Select PSU Seeds

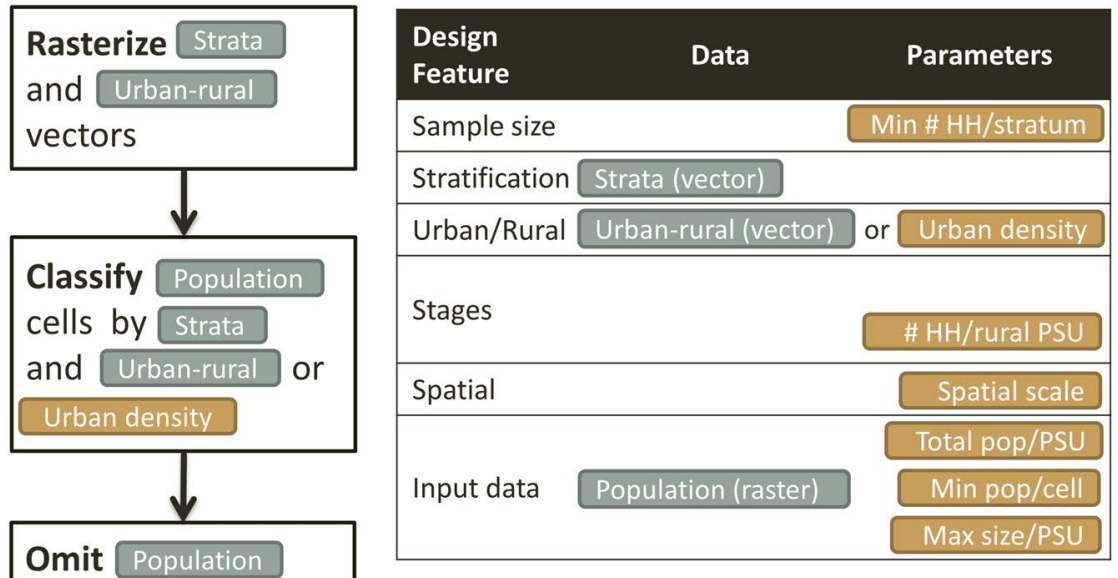

Generate PSU Boundaries

cells with value

$<$ Min pop/cell

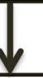

Randomly choose one seed cell

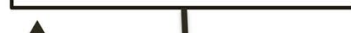

If urban or rural total households is $<$ Min. \# HH/strata, then sample a cell from the smaller domain, dropping a cell from the larger domain in the same stratum

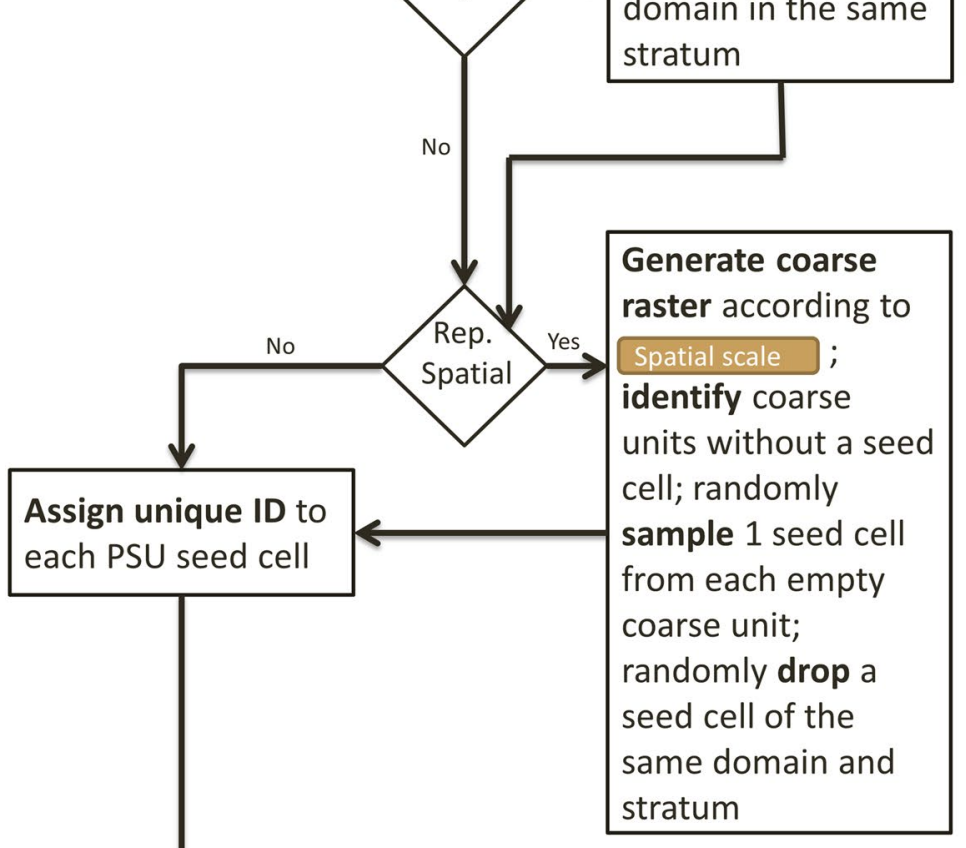

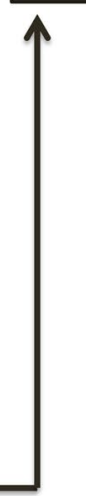
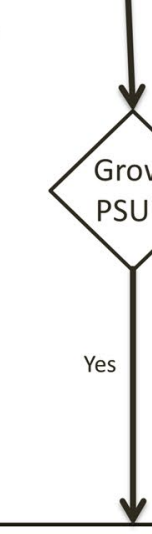

Calculate distance from seed cell to its neighbors; choose nearest neighbor cell and add population to $\mathrm{PSU}$

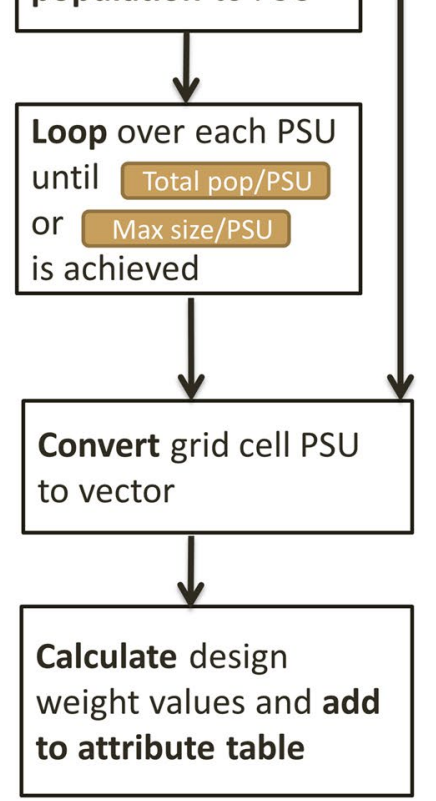

Fig. 2 GridSample workflow 
Table 1 Summary of attributes in the output shapefile

\begin{tabular}{|c|c|c|}
\hline Label & Type & Description \\
\hline PSUid & Integer & PSU identifier \\
\hline stratum & Integer & Stratum identifier \\
\hline psu_pop & Decimal & $\begin{array}{l}\text { Estimated population in PSU derived by } \\
\text { summing the seed cell and any growth } \\
\text { cells selected for PSU }\end{array}$ \\
\hline psu_r_pop & Decimal & $\begin{array}{l}\text { Estimated rural population in PSU derived } \\
\text { by summing all rural cells selected for } \\
\text { PSU }\end{array}$ \\
\hline psu_u_pop & Decimal & $\begin{array}{l}\text { Estimated urban population in PSU } \\
\text { derived by summing all urban cells } \\
\text { selected for PSU }\end{array}$ \\
\hline psus_in_stratum & Integer & Number of PSUs in the stratum \\
\hline str_pop & Decimal & $\begin{array}{l}\text { Estimated population in stratum derived } \\
\text { by summing all grid cells }\end{array}$ \\
\hline str_r_pop & Decimal & $\begin{array}{l}\text { Estimated rural population in stratum } \\
\text { derived by summing all grid cells classi- } \\
\text { fied as rural }\end{array}$ \\
\hline str_u_pop & Decimal & $\begin{array}{l}\text { Estimated urban population in stratum } \\
\text { derived by summing all grid cells classi- } \\
\text { fied as urban }\end{array}$ \\
\hline str_cells & Integer & Number of total cells in the stratum \\
\hline xCent & Decimal & $\begin{array}{l}\text { Longitude of PSU seed cell centroid in } \\
\text { decimal degrees }\end{array}$ \\
\hline yCent & Decimal & $\begin{array}{l}\text { Latitude of PSU seed cell centroid in } \\
\text { decimal degrees }\end{array}$ \\
\hline U_R & Character & $\begin{array}{l}\text { Urban or rural label based on whether the } \\
\text { seed cell was classified as urban or rural }\end{array}$ \\
\hline
\end{tabular}

reproduce an existing sample. The following attributes are needed to calculate sample weights (presented later): number of selected PSUs in stratum (psus_in_stratum), estimated population in stratum (str_pop), and estimated population in PSU (psu_pop).

\section{GridSample: clustered sampling}

GridSample supports the first-stage of the typical twostage cluster design used by DHS, MICS, and LSMS, as well as several other common survey designs. The user defines the desired total population in each PSU ( $\mathrm{cfg}$ pop_per_psu), ranging from 400 to 600 people in typical household surveys. Alternatively, GridSample can be used to select one-stage cluster samples by setting the total population per PSU (cfg_pop_per_psu) equal to the number of households to be sampled per PSU (cfg_hh_per_urban and cfg_hh_per_rural) multiplied by the average household size (available from previous surveys). Likewise, GridSample might be used to select a random sample of households by setting total population per PSU ( $\mathrm{cfg}$ pop per psu) equal to the average household size, and setting the number of households to be sampled per PSU (cfg_hh_per_urban and cfg_hh_per_rural) equal to 1. To implement a random sample of households, the user would additionally need to use a method to identify a random dwelling within each PSU [8].

\section{GridSample: stratification}

Strata should be mutually exclusive geographic areas that cover the entire population. In typical household surveys, sub-national administrative areas such as provinces or districts serve as strata, and sometimes these areas are further stratified into rural and urban areas. Independent samples will be selected from each stratum allowing strata-level estimates to be compared after the survey. While some gridded population datasets provide estimates of population by age-group and sex $[25,52,53]$ or household poverty level [54, 55], GridSample does not currently include a mechanism for non-geographic stratification, though the user could, in principal, sample from gridded population datasets of social-demographic groups.

To generate a geographically stratified sample in GridSample, the user defines strata boundaries with strata raster, and specifies the sample size per stratum with cfg_hh_per_stratum. This means that if the national sample size is 10,000 households from 5 provinces, then cfg_hh_per_stratum $==2000$. If the survey were additionally stratified by urban/rural such that there are 10,000 households sampled from 10 strata, then strata raster should include the boundaries of both urban/rural areas and provinces, and cfg_hh_per_stratum $==1000$.

\section{GridSample: urban/rural oversampling}

If urban/rural populations are not stratified, they may instead be treated as sub-domains. Sub-domains represent important sub-populations for which representative statistics are generated from the survey data, and thus each sub-domain should meet the minimum stratum sample size requirement (cfg hh per stratum). If either the urban or rural sub-domain does not include enough households, then the algorithm uses the ordered data frame to choose the next cell from the under-represented sub-domain (from any strata) and swaps out an already selected seed cell of the opposite sub-domain within that stratum. This process repeats until the sample size requirement is met in each sub-domain (cfg_hh per_stratum). To implement sub-domain representation in gs_sample, set cfg_sample_rururb $==1$ and define urban/rural boundaries (urban raster).

In practice, rural areas may be more difficult and expensive to visit, and thus a greater number of households might be sampled from rural PSUs than urban PSUs. This is why the user may specify different numbers 
of households to be sampled from urban PSUs (cfg_hh per_urban) and rural PSUs (cfg_hh_per_rural). If the same number of households will be sampled from all PSUs, then the user only needs to specify households to be sampled from urban PSUs (cfg_hh_per_urban).

\section{GridSample: spatial oversampling and other features}

Oversampling in space is analogous to oversampling urban/rural sub-domains. To select a sample that is both representative of the population and of space in GridSample, set cfg_sample_spatial $==1$ and specify the spatial scale (in square kilometers) at which the sample should be representative (cfg_sample_spatial_scale). For example, cfg_sample_spatial $\mathrm{scale}==20$ means that a coarse grid system with cells $20 \mathrm{~km} \times 20 \mathrm{~km}$ will be overlaid on the study area. If a coarse grid cell does not contain a PSU seed cell, then the first cell within the serpentine ordered data frame located inside the course cell will be selected, and another seed cell from the same stratum and sub-domain will be randomly dropped. To overcome the issue of slightly smaller grid cells toward the poles, GridSample calculates the area of the centroid (geographic center) grid cell in the study area, and uses that average grid cell size to generate the coarse grid with the correct dimensions.

The spatial scale of the survey is ideally linked to the scale of planned small area estimates. For example, if the sample is stratified by province (level 1 administrate units), and small area estimates will later be generated for districts (level 2 administrative units), then the median size of districts could be used. Determining an appropriate spatial scale may take trial and error. If the country has large areas of sparse population, the user might need to (a) increase the size of the spatial scale (cfg_sample_spatial_scale), or (b) force the algorithm to generate more PSUs in each stratum by increasing the sample size per stratum (cfg_hh per_stratum) and/or reduce the number of households sampled in each PSU (cfg_hh_per_urban and cfg_hh_per_rural).

GridSample offers several additional parameters. (1) The user can input a $100 \mathrm{~m} \times 100 \mathrm{~m}$ gridded population dataset, and then aggregate cells for the sample frame (e.g. $300 \mathrm{~m} \times 300 \mathrm{~m}$ sample frame cells would be generated by setting cfg_desired_cell_size = 3). Aggregating gridded population estimates usually increases the accuracy of each grid cell. Note that guidance regarding the ideal cell size of gridded population sample frames is not yet available. Other parameters include: (2) minimum population per cell (cfg_min_pop_per_cell) which will exclude grid cells from the sample frame with less than the specified minimum population, (3) maximum area of the PSU in squared kilometers (cfg_max_psu size) to ensure that PSUs can be feasibly enumerated during fieldwork, (4) random number value (cfg_random_number) to reproduce a previous gridded population sample, and (5) halt the PSU growth process (cfg_psu growth $=$ FALSE) discussed in detail below.

\section{Results}

We replicated the first-stage sample of the 2010 Rwanda DHS in GridSample. The 2010 Rwanda DHS sampled 12,540 households from 492 PSUs comprising rural villages and urban neighborhoods [56]. The sample was stratified by Rwanda's 30 districts, urban areas were oversampled by adding 12 PSUs in Kigali's three districts, and 26 households were sampled from each urban and rural PSU. The average village in Rwanda had 610 occupants according to the sample frame of 14,837 villages/neighborhoods. To replicate the 2010 Rwanda DHS in GridSample, we loaded the GridSample package, the raster package to prepare the data for GridSample, and set a working directory:

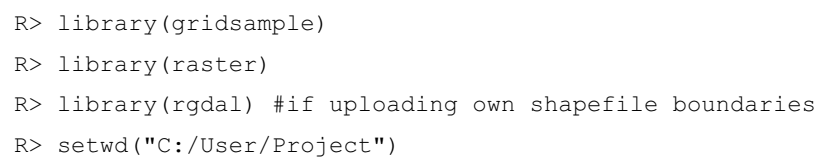

Next, we called the Rwanda 2010 UN-adjusted gridded population estimates preloaded in GridSample and also available at the WorldPop website [33]. This dataset was generated from 2002 Rwanda Census block data and 15 spatial covariates using a random forest model with dasymetric redistribution as described in the metadata [57] and cited methods paper [30].

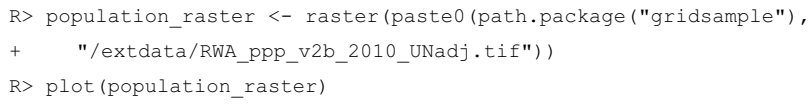

Then we loaded an unprojected shapefile of Rwanda's 30 district boundaries to use as strata. This file is preloaded in GridSample, and can be downloaded from MapLibrary [50]. We rasterized strata boundaries using the WorldPop population raster dimensions and assigned strata ID (ADM2_ID) as the grid cell value (numeric district identifier values ranged from 1 to 30): 


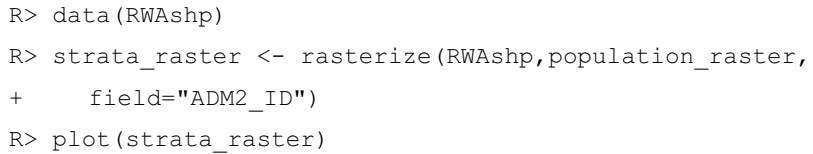

We considered using GUF, Modis or GRUMP to distinguish urban and rural areas, though we decided that these global models were not well suited for the largely rural context of Rwanda [38]. Instead, we calculated a sensible value to distinguish rural and urban cells directly from the WorldPop population raster. According to the 2012 Census, the National Institute of Statistics in Rwanda classifies $16 \%$ of the population as urban [58]. Thus, we identified the cell density value associated with $16 \%$ of the population living in the most populous cells, and used that value (11 people per $100 \mathrm{~m} \times 100 \mathrm{~m}$ cell) to create a binary raster of urban areas (value 1) and rural areas (value 0 ).

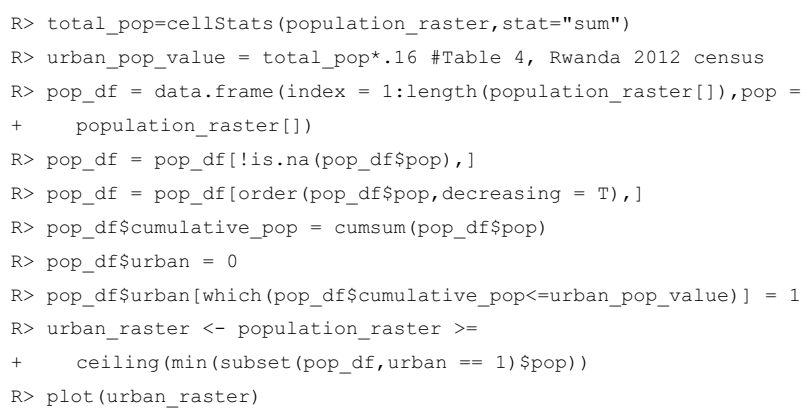

Note that the value used to differentiate urban and rural cells was found with the following code.

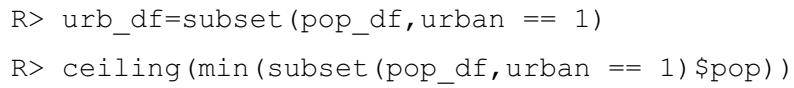

The gridded population, rasterized strata, and rasterized urban/rural layers are visualized in Fig. 3. We used these input data, plus parameters for total household sample size per stratum (cfg hh per stratum = 416), grow PSUs (cfg_psu_growth $=$ TRिU) to a minimum population total per PSU (cfg_pop_per_psu = 610), and household sample size per urban and rural PSU (cfg hh_per_urban $=26$ and cfg_hh_per_rural $=26$ ), to generate a gridded population sample with the same design as the 2010 DHS. We prevented sampling of cells with very small probability of population (cfg_min pop_per_cell $=0.01)$, limited the PSU size to $10 \mathrm{~km} \times 1 \overline{0} \mathrm{~km}(\mathrm{cfg} \max \mathrm{psu}$ size $=10)$, and specified the name (sample name = "rwanda psu sam$\mathrm{ple}$ ") and file location (output_path $="$ " $\mathrm{C}: / \bar{U}$ ser/ Project/data") to save the output shapefile.

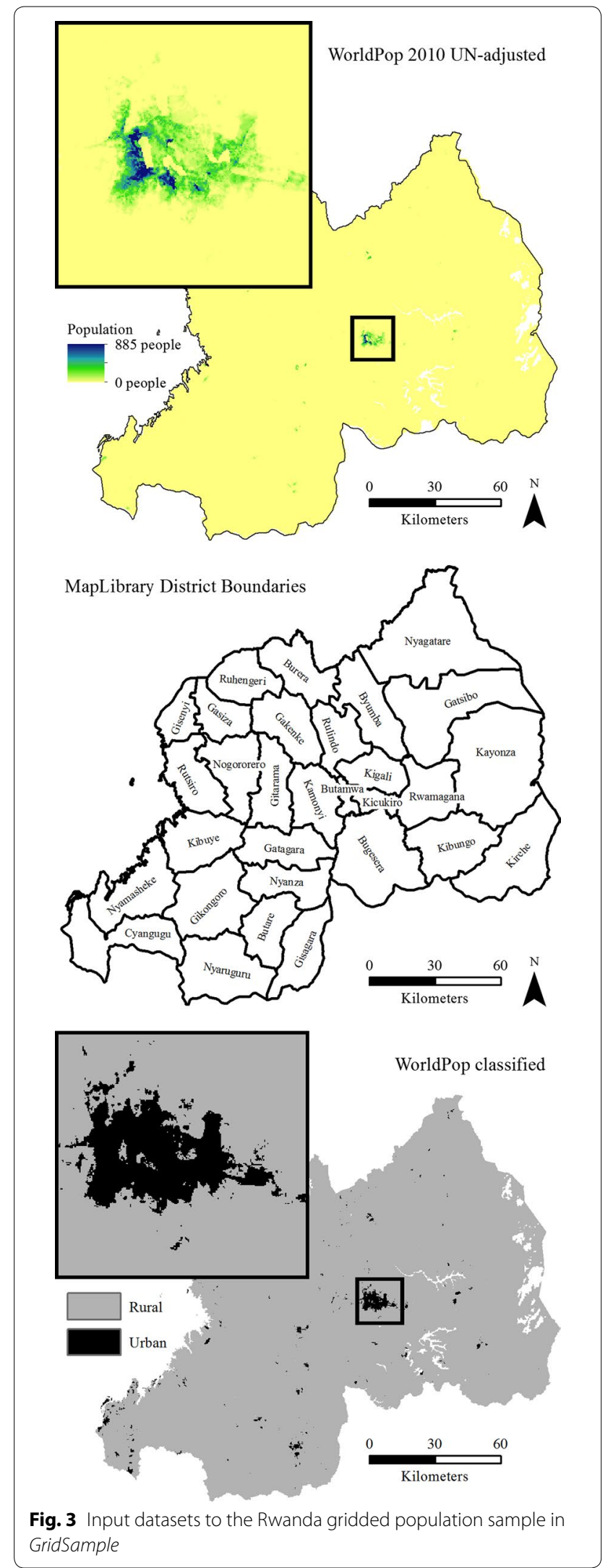




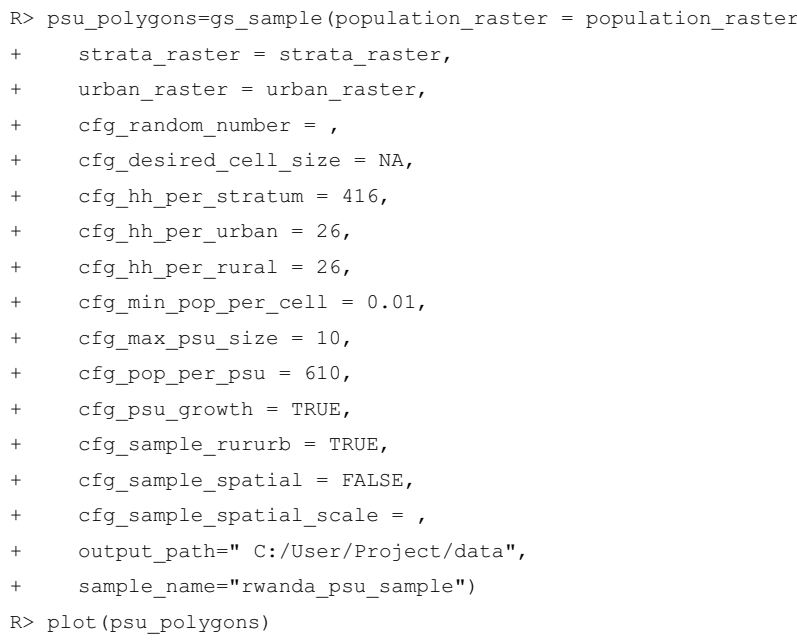

The Rwanda DHS selected 79 urban PSUs and 413 rural PSUs from their census sample frame. GridSample produced a similar sample of 75 urban PSUs and 405 rural PSUs (Table 2) which followed a similar geographic pattern as the Rwanda DHS (Fig. 4) using the WorldPop sample frame. In the GridSample-generated sample [59], the mean population per PSU was 620 people with one outlier that had 1479 people, and the median population was 612 people per PSU. One key difference between the samples was that the DHS added PSUs during the oversample, while GridSample re-distributed PSUs during the oversample, resulting in fewer PSUs. A second key difference was that DHS purposefully oversampled in the Kigali metropolitan area (Gasabo, Kicukiro and Nyarugenge districts) while GridSample oversampled from all urban areas, including smaller cities in Gisenyi, Cyangugu, and Gikongoro districts.

\section{Discussion}

Gridded population sampling methods are in their infancy. Several approaches to first-stage sample selection and to fieldwork have been tried. These approaches are promising but have limitations and require further research. The GridSample $\mathrm{R}$ algorithm provides a tool to develop and evaluate emerging gridded population sampling methods.

\section{Modifiable Areal Unit Problem}

Gridded population sampling is sensitive to the modifiable areal unit problem (MAUP). A MAUP emerges when an arbitrary spatial unit, such as a grid cell, is used to summarize continuous population characteristics leading to apparently different spatial patterns of that characteristic in the population simply by changing the size (scale) or zone (grouping) of the spatial units [60]. In
Table 2 Number of primary sampling units in a Demographic and Health Survey and equivalent GridSample survey

\begin{tabular}{|c|c|c|c|c|c|}
\hline \multirow[t]{2}{*}{ District name } & \multirow[t]{2}{*}{ Alternative name } & \multicolumn{2}{|l|}{ DHS } & \multicolumn{2}{|c|}{ GridSample } \\
\hline & & Urban & Rural & Urban & Rural \\
\hline Bugesera & Bugesera & & 16 & 2 & 14 \\
\hline Burera & Burera & & 16 & 1 & 15 \\
\hline Butamwa & Nyarugenge & 19 & 1 & 15 & 1 \\
\hline Butare & Huye & 3 & 13 & 3 & 13 \\
\hline Byumba & Gicumbi & 2 & 14 & 1 & 15 \\
\hline Cyangugu & Rusizi & 2 & 14 & 5 & 11 \\
\hline Gakenke & Gakenke & & 16 & & 16 \\
\hline Gasiza & Nyabihu & & 16 & 1 & 15 \\
\hline Gatagara & Ruhango & 3 & 13 & & 16 \\
\hline Gatsibo & Gatsibo & & 16 & & 16 \\
\hline Gikongoro & Nyamagabe & 1 & 15 & 3 & 13 \\
\hline Gisagara & Gisagara & & 16 & & 16 \\
\hline Gisenyi & Rubavu & 1 & 15 & 10 & 6 \\
\hline Gitarama & Muhanga & 4 & 12 & 1 & 15 \\
\hline Kamonyi & Kamonyi & & 16 & & 16 \\
\hline Kayonza & Kayonza & & 16 & & 16 \\
\hline Kibungo & Ngoma & 3 & 13 & 1 & 15 \\
\hline Kibuye & Karongi & 2 & 14 & 2 & 14 \\
\hline Kicukiro & Kicukiro & 20 & & 13 & 3 \\
\hline Kigali & Gasabo & 11 & 9 & 8 & 8 \\
\hline Kirehe & Kirehe & & 16 & & 16 \\
\hline Nogororero & Ngororero & & 16 & & 16 \\
\hline Nyagatare & Nyagatare & & 16 & & 16 \\
\hline Nyamasheke & Nyamasheke & & 16 & & 16 \\
\hline Nyanza & Nyanza & 4 & 12 & 2 & 14 \\
\hline Nyaruguru & Nyaruguru & & 16 & & 16 \\
\hline Ruhengeri & Musanze & 2 & 14 & 3 & 13 \\
\hline Rulindo & Rulindo & & 16 & 1 & 15 \\
\hline Rutsiro & Rutsiro & & 16 & & 16 \\
\hline Rwamagana & Rwamagana & 2 & 14 & 3 & 13 \\
\hline Total & & 79 & 413 & 75 & 405 \\
\hline
\end{tabular}

gridded population sampling, the size and zone of grid cells are likely to influence sampling inclusion probabilities, especially when the first-stage sample is based on geographically large grid cells, and/or the population is heterogeneously distributed.

Four general approaches to first-stage sampling with gridded population data are outlined in Fig. 5. First, the segmentation approach involves sampling geographically large PSUs with probability proportionate to estimated population size, then segmenting by smaller grid cells [10] or manually delineate smaller areas using satellite imagery [6-10]. GridSample can be used to select large cells by aggregating the input gridded population dataset. In Myanmar, Muñoz and Langeraar (2013) 


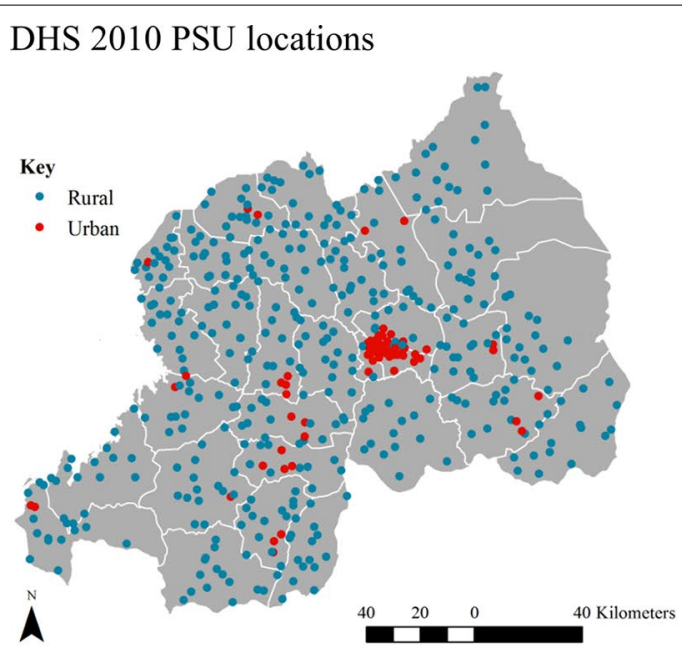

Three example DHS urban PSU coordinates, with $2 \mathrm{~km}$ buffers showing possible range of geodisplacement, and close up of imagery

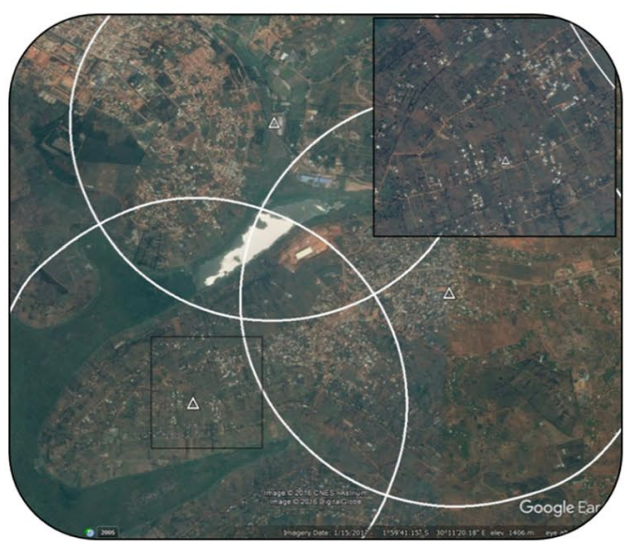

Three example DHS rural PSU coordinates, with $5 \mathrm{~km}$ buffers showing possible range of geodisplacement, and close up of imagery

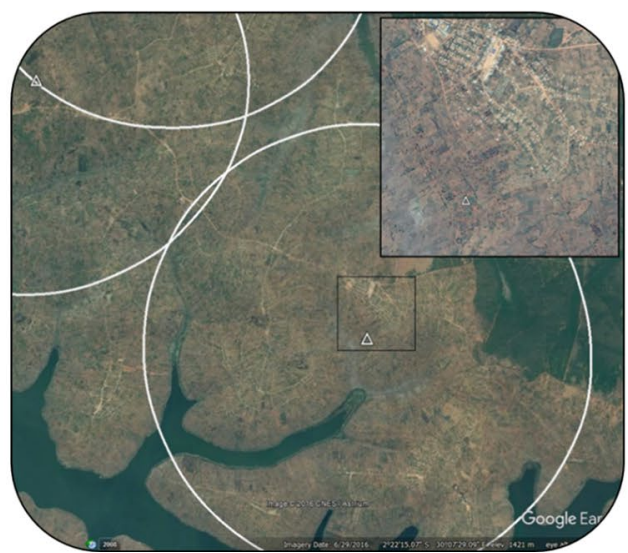

GridSample 2010 PSU locations

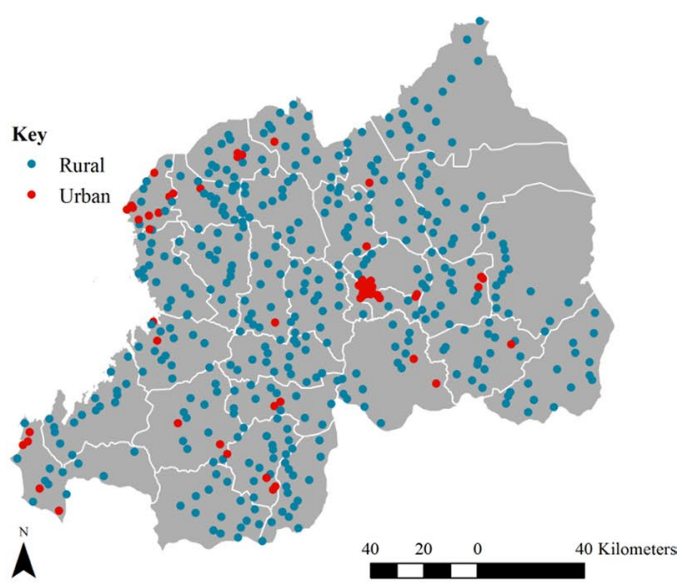

One example GridSample urban PSU boundary and its corresponding seed cell coordinate

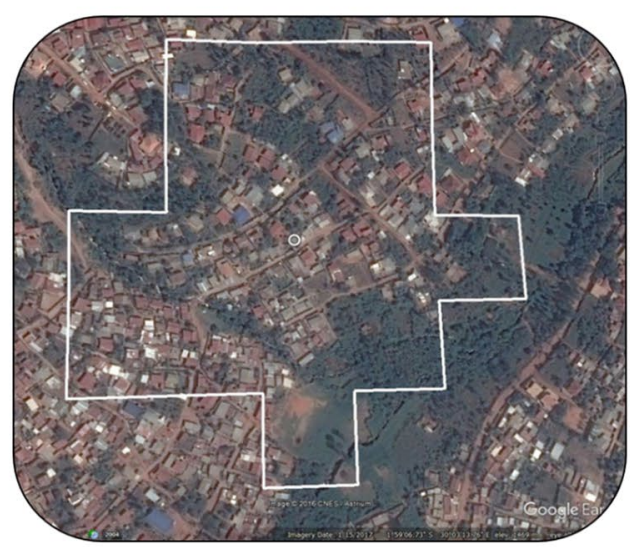

One example GridSample rural PSU boundary and its corresponding seed cell coordinate

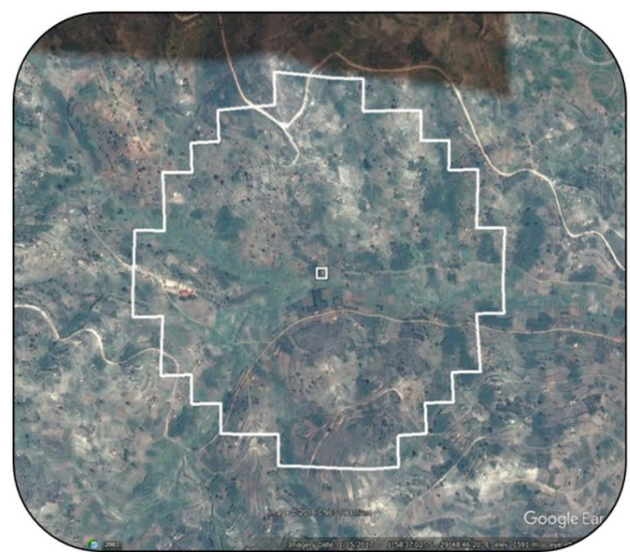

Fig. 4 Visual comparison of primary sampling units (PSUs) generated by the 2010 Rwanda DHS [56] and GridSample 


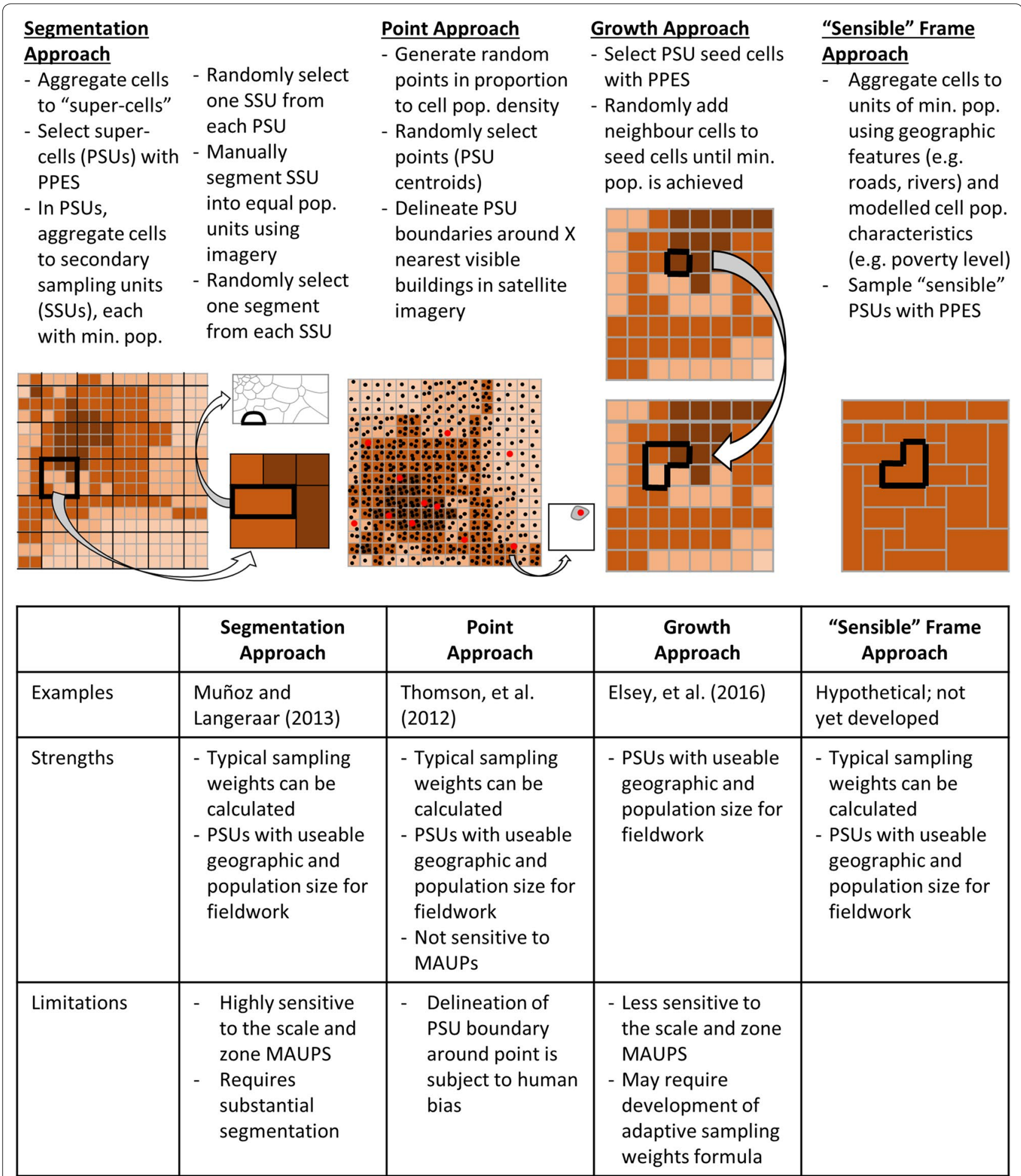

Fig. 5 Probability sampling approaches with gridded population data

aggregated LandScan $1 \mathrm{~km} \times 1 \mathrm{~km}$ gridded population estimates to $3 \mathrm{~km} \times 3 \mathrm{~km}$ "super" cells for selection of the first-stage sample. Then they grouped $1 \mathrm{~km} \times 1 \mathrm{~km}$ grid cells within the selected PSUs to meet a minimum population threshold, and then randomly sampled one group of cells as a secondary sampling unit (SSU) in each PSU. Finally, they manually segmented SSUs into dozens of areas with roughly equal population based on satellite 
imagery, and sampled one segment [10]. As a result, the sample weights were computationally straightforward to calculate because they followed a typical multi-stage sampling approach. Additionally, the final sampling units had sensible boundaries related to features in the real world, making fieldwork feasible. However, sample inclusion probabilities of PSUs and SSUs were sensitive to the size and zone of grid cells, which could have smoothedout or emphasized population density depending on the distribution of the underlying population.

A point approach was used by Thomson and colleagues (2012) using LandScan $1 \mathrm{~km} \times 1 \mathrm{~km}$ gridded population data in the eastern D. R. Congo. For this survey, the team generated randomly located points within grid cells where the number of points was proportional to estimated population. Then they randomly sampled points within strata. Finally, they manually delineated sampling units around the nearest dwellings to each point using satellite imagery, ensuring that PSU boundaries were located within cell boundaries [6]. Sample weights were adapted to follow a typical multi-stage sampling approach, the final sampling unit boundaries were sensible, making fieldwork feasible, and the use of points prevented any effect of the MAUP. However, the manual delineation of one sampling unit around each point was subject to human bias.

The third approach to gridded population sampling is the growth approach, uniquely available in the GridSample tool. Elsey et al. [7] in Kathmandu, Nepal used an early version of GridSample to select seed cells from WorldPop's $100 \mathrm{~m} \times 100 \mathrm{~m}$ gridded population dataset, and grew PSUs to a minimum population size. Growing PSUs is likely less sensitive to the zone and scale MAUPs than segmenting large cells because, in the growth approach, the scale of the starting grid cells is closer in geographic and population size to the final sampling unit. However, the correct calculation of sampling inclusion probability weights for the growth approach is unclear. Should sample probability weights be calculated from the grid cell densities, or the densities of final sampling units? Arguments can be made for both approaches. Before discussing two potential sample weight calculations for the growth approach, we describe a hypothetical, but feasible, fourth approach to gridded population sampling.

Perhaps the most ideal gridded population sample frame would group grid cells into "sensible" potential PSUs of similar population size before first-stage sampling. Sensible PSU boundaries would be defined in terms of geographic features such as roads, rivers, ridges or valleys that could be easily recognized and navigated in the field. Sensible PSUs would also group similar types of populations, for example, by grid cell mean poverty level. Generation of a sensible gridded population sample frame has only recently become possible as new techniques are developed to estimate population characteristics, such as poverty-level or disease status, in a gridded population format [54,61]. The use of quadtree methods to divide dense population grid squares into four smaller cells can be viewed as a rudimentary first step toward development of sensible potential PSUs [62]. If a gridded population sample frame of sensible potential PSUs existed, the survey practitioner would sample units with probability proportionate to estimated size, and calculate typical sampling inclusion probability weights.

The growth approach to gridded population sampling may be conceptualize of as one instance of a sensible frame in which only the boundaries of the sampled PSUs are known, and the boundaries of non-sampled potential PSUs exist but are not drawn. Sample weights calculated from the final PSU population densities are straightforward to calculate, and are provided below.

If, however, the growth approach inclusion probabilities need be calculated from grid cell (rather than final PSU) population densities, then a complex adaptive sample weight needs to be formulated [63]. An adaptive sample weight would account for the estimated population of a given cell, as well as the probability of being grown into a PSU via a neighboring cell. The probability of being grown into a PSU would depend on (a) the estimated populations of neighboring cells, (b) the parameter for PSU maximum geographic size, (c) the parameter for PSU minimum population size, and possibly (d) the location of strata boundaries, and (e) the location of voronoi polygon boundaries between seed cells in a multitude of sample instances. The need for such a complex formulation needs to be evaluated, but is beyond the scope of this paper.

\section{Sample weights}

Below, we provide sample weight calculations for the growth approach to PSU selection, which is uniquely available in GridSample. These weights reflect inclusion probabilities in the final PSUs, and not of individual grid cells. Sample weights for the segmentation, point, and sensible PSU approaches have been described elsewhere and are summarized in Additional file 1. These sample weight formulations parallel typical survey methods, reflecting the probability that a household is (1) selected, (2) found, and (3) responded [13-16]. While the GridSample output shapefile includes values needed to calculate PSU selection probabilities, the survey implementer must track the number of households enumerated in the field in each PSU, and household response rates to correctly calculate sample weights. The following formulas use four indices: $1 . . . k$ strata, $1 . . . i$ PSUs, $1 . . . j$ households, and $1 \ldots q$ individuals. The household selection (base) weight for the growth approach to PSU formation-the 
probability that PSU $i$ is selected, and then household $j$ is selected-is given by:

$$
w_{i j . b}=\frac{1}{P_{i} \times P_{j(i)}}=\frac{G_{k} / g_{i k}}{n_{k}} \times \frac{M_{i k}}{m_{i k}}
$$

where $n_{k}$ is the number of selected PSUs in stratum $k, G_{k}$ is the estimated total population in stratum $k, g_{i k}$ is the estimated population in PSU $i$ in stratum $k, m_{i k}$ is the number of households sampled in PSU $i$ and stratum $k$ during fieldwork, and $M_{i k}$ is the number of total households enumerated in PSU $i$ and stratum $k$ during fieldwork.

If growth PSUs are manually divided and further sampled, weights are calculated in the same way, except that the probability of being in the final sample unit $w_{i j . b}$ includes $b_{i k}$, the proportion of households located in the manually-drawn segment, approximated by counting buildings in satellite imagery:

$$
w_{i j . b}=\frac{G_{k} / g_{i k}}{n_{k}} \times \frac{M_{i k}}{m_{i k}} \times \frac{1}{b_{i k}}
$$

The household response weight-the probability that PSU $i$ is found and sampled, and household $j$ is found and responded-is given by:

$$
w_{i j . r}=\frac{1}{P_{i . r} \times P_{j . r(i)}}=\frac{n_{k}}{n_{k} *} \times \frac{m_{i k}}{m_{i k} *}
$$

where $n_{k}$ number of selected PSUs in stratum $k, n_{k} *$ is the number of found and sampled PSUs in stratum $k, m_{i k}$ is the number of selected households in PSU $i$ and stratum $k$, and $m_{i k} *$ is the number of found and responded households in PSU $i$ and stratum $k$. The individual response weight-the probability that PSU $i$ is found and sampled, then household $j$ is found and responds, and finally that individual $q$ is present and responds-is given by:

$$
w_{i j q . r}=\frac{1}{P_{i . r} \times P_{j . r(i)} \times P_{q . r(j i)}}=\frac{n_{k}}{n_{k} *} \times \frac{m_{i k}}{m_{i k} *} \times \frac{u_{i j k}}{u_{i j k} *}
$$

where $n_{k}$ is the number of selected PSUs in stratum $k, n_{k} *$ is the number of found and sampled PSUs in stratum $k$, $m_{i k}$ is the number of selected households in PSU $i$ and stratum $k, m_{i k} *$ is the number of found and responded households in PSU $i$ and stratum $k$, and $u_{i j k}$ is the number of eligible individuals in household $j$ in PSU $i$ and stratum $k$, and $u_{i j k} *$ is the number of responded individuals in household $j$ in PSU $i$ and stratum $k$. The household sample weight $w_{i j}$ is comprised of the household selection weight and household response weight:

$$
w_{i j}=w_{i j . b} \times w_{i j . r}
$$

Assuming that all eligible individuals (e.g., all women age 15-49) will be interviewed in the selected households, the individual sample weight $w_{i j q}$ is comprised of the household selection weight and individual response weight:

$$
w_{i j q}=w_{i j . b} \times w_{i j q . r}
$$

\section{Fieldwork}

Four approaches are available for survey fieldwork with GridSample output. These four approaches are visualized in Fig. 6, and described below.

\section{Gridded PSUs}

This option uses gridded PSU boundaries which have squared corners and no relation to geographic or administrative features in the real world. This approach was used in a two-stage cluster survey of households in Kathmandu, Nepal [7]. The team used OpenStreet$\mathrm{Map}^{\mathrm{TM}}$, a crowd sourced online map of roads, building locations, and other features, via an Android application on mobile phones to digitally map households within PSUs. OpenStreetMap ${ }^{\mathrm{TM}}$ enumeration was chosen over typical pen-and-paper mapping, in part, because half of their PSUs were already mapped in OpenStreetMap ${ }^{\mathrm{TM}}$. Households (defined as a group of people who share a cook pot) were fully enumerated by knocking on doors and talking to neighbors ensuring that lower-income households who shared an apartment were not undersampled. The team encountered, sometimes substantial, differences in the number of households per PSU than were expected from the WorldPop sample frame, so they planned to interview every $10^{\text {th }}$ household regardless of PSU size to achieve a probability sample. The team cited geographic accuracy in field maps, feasibility of mapping in dense, complex urban environments, leveraging of existing data, and the ability to contribute to a crowd-sourced resource as reasons to use this approach [7].

We support the use of OpenStreetMap ${ }^{\mathrm{TM}}$ enumeration, especially for urban settings where OpenStreet$\mathrm{Map}^{\mathrm{TM}}$ data are likely to exist. However, we strongly recommend that implementers employ a method to anonymize buildings added to the crowd-sourced map such that interviewed PSUs cannot be identified. In areas where buildings have already been mapped in OpenStreetMap $^{\mathrm{TM}}$, minor edits will not reveal PSU locations. However, in areas of the map without building and road locations, implementers should consider mapping beyond the edges of the PSU boundaries so that gridded PSU shapes do not suggest a gridded household survey. Furthermore, if OpenStreetMap $^{\mathrm{TM}}$ data are sparse in the survey region, implementers should consider enumerating a number of fake PSUs to preserve the anonymity of interviewed communities. Specific guidelines for OpenStreetMap $^{\mathrm{TM}}$ enumeration are not yet available. 


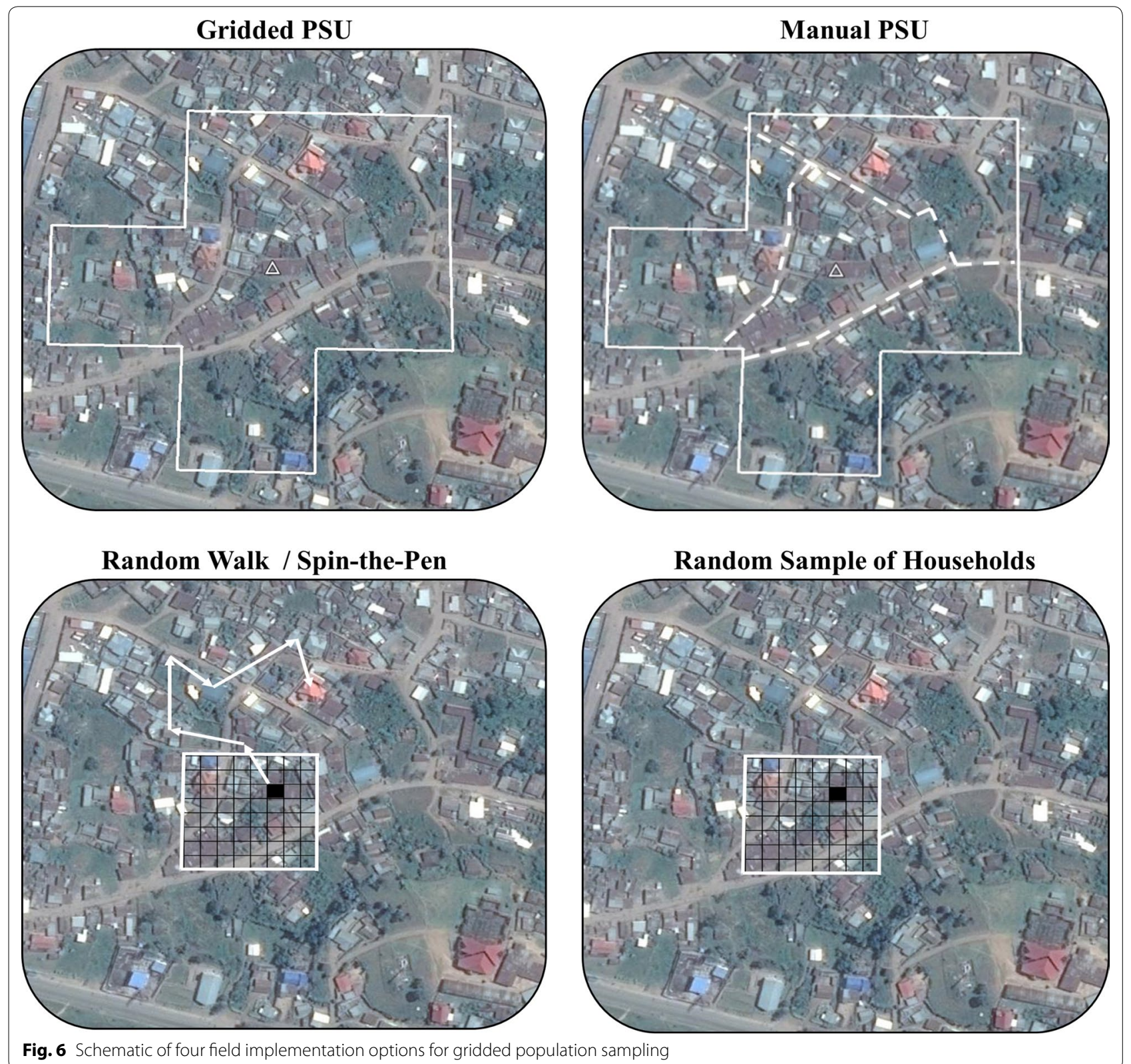

\section{Manually-drawn PSUs}

A second approach to implement gridded population samples is to manually draw PSUs around random points within seed cells, or to manually segment gridded sampling units using detailed satellite imagery. Manuallydrawn PSUs were used in a one-stage cluster survey in eastern D. R. Congo [6] and a two-stage cluster survey in Myanmar [10]. A key benefit of this approach is that PSUs follow sensible boundaries such as rivers and roads, which are easily identified in both satellite images and in the field. Because manually-drawn PSUs are easily identifiable, field teams are flexible to use hand-sketched pen and paper maps, printed maps of satellite imagery or OpenStreetMap $^{\mathrm{TM}}$ features, or digital maps for field navigation and household enumeration.

\section{Non-probability samples}

Random-walk and "spin-the-pen" sampling methods result in non-probability samples of the population and are thus not recommended by surveyors [64-66]. Nonetheless, these and similar methods are often used in rapid or high-security field assessments because they are cheaper and faster to implement than typical two-stage cluster samples. Random-walk and spin-the-pen gridded 
sampling methods were used in rapid assessments in Iraq [8] and Myanmar [11]. In both studies, gridded population datasets were considered to be more accurate sample frames than other available population data. Because random-walk and spin-the-pen methods do not lead to probability samples, we do not provide sample probability weights.

\section{Simple random sample of households}

Researchers sometimes perform simple random samples of households in small study areas-for example, a refugee camp or a single city-by digitizing dwelling point locations in a satellite image and sampling points at random [67-71]. While a simple random sample of households has not been conducted using gridded population sampling, it would be straightforward to implement. Grid cells would be sampled with probability proportionate to estimated size, and the growth algorithm could optionally be switched off to generate single cell PSUs. Then a single dwelling would be randomly chosen within selected cells, either from mapping all dwellings or using a method like the one described by Galway and colleagues in Iraq [8]. In the Iraq study, the team overlaid a $10 \mathrm{~m} \times 10 \mathrm{~m}$ minigrid on Google Earth ${ }^{\mathrm{TM}}$ satellite imagery within the seed cell, and then randomly selected one mini-grid unit. If the $10 \mathrm{~m}$ mini-grid unit covered a building, the building was selected for sampling, otherwise the process was repeated until the first building was randomly identified in the imagery. If the randomly selected building had multiple households or was non-residential, one nearby household could be randomly selected as describe by Siri and colleagues in Kenya [66]. A simple random sample of households would not require sample weights.

\section{Limitations}

Gridded population data are increasingly used as an alternative survey sample frame in countries where census data are outdated or inaccurate. Gridded population sample frames may also be used in lieu of census data for surveys that need to be representative of both population and of space, and where PSUs of a specific population size are needed. Next we discuss six areas where research is underway, or needed, to address limitations of gridded population sampling.

\section{Accuracy of gridded population sample frame}

The first major concern in gridded population sampling is the accuracy of the underlying gridded population data. Gridded population sampling has been tried by a number of survey implementers in circumstances of outdated or inaccurate census data, however the accuracy of gridded population datasets are varied, and often unquantified. Accuracy of publically available top-down gridded population data is dependent on several model components: (1) accuracy of the input census data, (2) the geographic scale of the input census data (e.g. census tract-level versus district-level), (3) the age, accuracy, and type of model covariate data, and (4) the model algorithm itself. The geographic scale of the output grid also matters for measurement of accuracy; grid cell estimates in a $1 \mathrm{~km} \times 1 \mathrm{~km}$ gridded population dataset will almost always be more accurate than grid cells in a $100 \mathrm{~m} \times 100 \mathrm{~m}$ gridded population dataset. Model errors are difficult to estimate, and to even conceptualize, for gridded population datasets that rely on simple disaggregation approaches, as they are essentially gridded representations of the input census data [24]. While prediction errors can be calculated for gridded population datasets derived from complex modelling techniques, WorldPop is the only dataset to include errors [see, for example, 56]. However, it is unclear how survey implementers can use prediction errors to quantify or improve the accuracy of household survey sample frames.

Numerous studies have evaluated the accuracy of gridded population estimates against ground-collected settlement locations [72], against census data available at a finer-scale than the census data used in the model [29, 73-76], and by comparing old and new gridded population datasets where the new dataset uses updated or finer-scale population data [38]. Still this evidence is not sufficient to assess the accuracy of a specific top-down gridded population dataset. Given the number of components that contribute to gridded population model error, future research should utilize simulation studies to test the effects of various model components on gridded population estimates. These studies should also reframe how the estimate errors are addressed (e.g. rather than ask "how much error is there around the estimate for each cell of size X?", researchers should ask "how many cells need to be aggregated to achieve an error of Y?").

\section{Modifiable areal unit problem}

Second, segmentation and growth approaches to sample unit selection might be subject to bias from the MAUP. Simulation studies should be used to quantify the effects of grid cell sizes and groupings on PSU selection probabilities. Additionally, development of geographically and socially sensible sample frames with gridded population data should be pursued. The ability to create a sensible gridded population sample frame is highly dependent on availability of fine scale, accurate environmental data and gridded estimates of population social-health characteristics.

\section{Adaptive PSU sample weights}

Third, where growth approaches are used for selection of PSUs, further research is needed to evaluate whether 
adaptive sample weights should be used, and if so, how to formulate them. These questions can be evaluated with statistical theory and simulation studies.

\section{Availability of satellite imagery}

Fourth, all of the approaches to gridded population sampling described here are dependent on access to fine-resolution satellite imagery with good visibility of dwellings without extensive tree-cover or cloud-cover. Existing gridded population samples have been implemented in cities, camps, deserts, savannah, and deforested farmlands; methods for implementing gridded population samples have not been described for forested areas.

\section{Concealing PSU locations in publications and crowd-sourced maps}

Fifth, gridded population samples that use crowd-sourced maps in fieldwork must guarantee anonymity of survey respondents and their communities. Crowd-sourced maps can be incredibly valuable for field navigation and household enumeration, though the technology and protocols to support survey activities are limited. Standard protocols have not yet been established to conceal survey PSU locations when mapping buildings and roads in a crowdsourced platform such as OpenStreetMap ${ }^{\mathrm{TM}}$. Furthermore, we are not aware of any applications that allow survey enumerators to both update OpenStreetMap ${ }^{\mathrm{TM}}$ and separately store a confidential household listing linked to building locations, which interviewers would need to identify sampled households. As in any survey, PSU boundaries and centroid point locations should not be shared publically to protect the anonymity of respondents and their communities. PSU point locations can be published if they are randomly geo-displaced following methods like those used by MeasureDHS [77]. The MeasureDHS project publishes PSU centroid coordinates that are displaced up to $2 \mathrm{~km}$ in urban areas, and up to $5 \mathrm{~km}$ in rural areas, with one in every 100th rural point displaced up to $10 \mathrm{~km}$.

\section{Fieldwork feasibility}

The sixth concern of gridded population sampling is feasibility of fieldwork. While there are multiple reasons to use gridded population sampling, protocols to use these methods in the field need further development. What is the enumeration protocol in a PSU that falls on two sides of a river where there is not a nearby bridge to cross? Should buildings be enumerated if they are intersected by the PSU boundary? Given that gridded PSU boundaries do not follow sensible geographic or administrative boundaries, recent satellite imagery is almost certainly needed during enumeration. What is the minimum image resolution required for sampling in rural versus urban areas? How recent should the satellite imagery be? What are the tradeoffs of using digital enumeration methods over paper-based methods? While the use of smart phones or tablets to digitally enumerate PSUs increases the cost and skill requirements among enumerators, it may also reduce the time and increase the accuracy of enumeration compared to pen-and-paper methods. Multiple issues related to cost, time, accuracy, technology, and staff skill requirements to implement gridded population surveys need to be evaluated.

\section{Conclusions}

The GridSample $\mathrm{R}$ package facilitates further research into the promising field of gridded population sampling. Gridded population sampling is an attractive alternative to typical sampling methods when census data are outdated or inaccurate. GridSample supports standard complex survey designs including clustered sampling, stratification, and oversampling in urban or rural areas. GridSample additionally allows users to oversample in space, and to specify a desired population size of sampling units. We show that GridSample can be used to replicate a DHS in Rwanda, providing evidence of a similar number of primary sampling units with similar population sizes in urban and rural areas. We also summarize four ways in which gridded population samples have been implemented in the field, and provide sample weight calculations for GridSample output. Finally, we discuss several areas of current and future research into gridded population sampling which can benefit from this tool.

\section{Additional file}

Additional file 1. Sample weight formulas for segmentation, point, and sensible PSU formation.

\section{Abbreviations}

CRAN: Comprehensive R Archive Network; DHS: Demographic and Health Survey; EA: Enumeration area; GHSL: Global Human Settlement Layer; GPW: Gridded Population of the World; GRUMP: Global Rural-Urban Mapping Project; GUF: Global Urban Footprint; LSMS: Living Standards Measurement Survey; MAUP: modifiable areal unit problem; MICS: Multiple Indicator Cluster Survey; PPES: probability proportionate to estimated size; PSU: primary sampling unit; SDG: sustainable development goals; SSU: secondary sampling unit; UNEP: United Nations Environment Programme.

\section{Authors' contributions}

DRT drafted the user requirements, conceptualized the first part of the GridSample algorithm related to PSU selection, tested the software, and wrote the first draft of the manuscript. FRS conceptualized the second part of the GridSample algorithm related to PSU growth and wrote the first draft of the $\mathrm{R}$ code. NWR refined the code and added to it conceptually, for example, by introducing voronoi polygons to ensure contiguous, non-overlapping PSUs. AJT facilitated team introductions, provided key technical guidance during the algorithm development, and contributed extensive revisions to the manuscript. MCC provided key practitioner guidance during the algorithm development, and contributed extensive revisions to the manuscript. All authors read and approved the final manuscript. 


\begin{abstract}
Author details
1 Department of Social Statistics and Demography, University of Southampton, Building 58, Southampton SO17 1BJ, UK. ${ }^{2}$ WorldPop, Department of Geography and Environment, University of Southampton, Building 44 Southampton SO17 1BJ, UK. ${ }^{3}$ Flowminder Foundation, Roslagsgatan 17, 11355 Stockholm, Sweden. ${ }^{4}$ Department of Geography and Geosciences, University of Louisville, 200 E Shipp Ave, Louisville, KY 40208, USA. ${ }^{5}$ Department of Global Health and Population, Harvard T.H. Chan School of Public Health, 665 Huntington Ave, Boston, MA 02115, USA.
\end{abstract}

\section{Acknowledgements}

Special thanks to Tomas Bird, Nikos Tzavidis, Shoaib Ali, Alesandro Sorichetta, Dale Rhoda, and Kristen Himelein who all provided helpful insights about sample weight calculations and spatial sampling. We also thank the two anonymous reviewers who asked questions and gave input that improved this manuscript.

\section{Competing interests}

The authors declare that they have no competing interests.

\section{Availability of data and materials}

The input data used in this analysis is available with the GridSample R package: Thomson DR, Ruktanonchai NW, Stevens FR, Castro M, Tatem AJ. 2017. gridsample: Tools for Grid-Based Survey Sampling Design. R package version 0.1.2. https://CRAN.R-project.org/package=gridsample. The output dataset from the GridSample R package is available online: Thomson DR. 2017. GridSample output: 2010 Rwanda DHS. Harvard Dataverse. http://dx.doi.org/10.7910/DVN/MSCJOD.

\section{Funding}

This work is supported by funding from the Bill \& Melinda Gates Foundation (OPP1 106427). DRT is supported by funding from the UK Economic and Social Research Council (Grant Number ES/J500161/1). FRS is supported by funding from the Bill and Melinda Gates Foundation (OPP1134076) with initial development supported by the National Science Foundation (0801544). NWR is supported by funding from Clinton Health Access Initiative. AJT is supported by funding from NIH/NIAID (U19A1089674), the Bill and Melinda Gates Foundation (OPP1106427, 1032350, OPP1134076, OPP1094793), the Clinton Health Access Initiative (which supports NR), National Institutes of Health, and a Wellcome Trust Sustaining Health Grant (106866/Z/15/Z). MCC thanks the support from the Department of Global Health and Population. The funders had no role in study design, data collection and analysis, decision to publish, and preparation of the manuscript.

\section{Publisher's Note}

Springer Nature remains neutral with regard to jurisdictional claims in published maps and institutional affiliations.

Received: 27 March 2017 Accepted: 4 July 2017

Published online: 19 July 2017

\section{References}

1. Global Health Data Exchange (GHDx). Institute for Health Metrics and Evaluation, Seattle. 2017. http://ghdx.healthdata.org/. Accessed 10 Mar 2017.

2. Global Health Observatory data repository. World Health Organization, Geneva. 2017. http://apps.who.int/gho/data/node.home. Accessed 10 Mar 2017.

3. Food Security Analysis: Assessments. World Food Programme, Rome. 2017. http://vam.wfp.org/?_ga=1.230081818.764469399.1485248139. Accessed 3 Mar 2017.

4. HDX Database v.1.8.3. Humanitarian data exchange. 2017 https://data humdata.org/. Accessed 10 Mar 2017.

5. Consumer Panels. Neilsen. 2017 http://www.nielsen.com/id/en/solutions/measurement/consumer-panels.html. Accessed 10 Mar 2017.

6. Thomson DR, Hadley MB, Greenough PG, Castro MC. Modelling strategic interventions in a population with a total fertility rate of 8.3: a cross-sectional study of Idjwi Island, DRC. BMC Public Health. 2012. doi:10.1186/1471-2458-12-959.
7. Elsey H, Thomson DR, Lin RY, Maharjan U, Agarwal S, Newell J. Addressing inequities in urban health: do decision-makers have the data they need? Report from the urban health data special session at international conference on urban health, Dhaka 2015. J Urban Health. 2016. doi:10.1007/ s11524-016-0046-9.

8. Galway L, Bell N, Sae AS, Hagopian A, Burnham G, Flaxman A, et al. A twostage cluster sampling method using gridded population data, a GIS, and Google EarthTM imagery in a population-based mortality survey in Iraq. Int J Health Geogr. 2012. doi:10.1186/1476-072X-11-12.

9. Hagopian A, Flaxman AD, Takaro TK, Esa Al Shatari SA, Rajaratnam J, Becker S, et al. Mortality in Iraq associated with the 2003-2011 war and occupation: findings from a national cluster sample survey by the University Collaborative Iraq Mortality Study. PLoS Med. 2013. doi:10.1371/ journal.pmed.1001533.

10. Muñoz J, Langeraar W. A census-independent sampling strategy for a household survey in Myanmar. 2013. http://winegis.com/images/censusindependent-GIS-based-sampling-strategy-for-household-surveys-planof-actionremoved.pdf. Accessed 10 Mar 2017.

11. Sollom R, Richards AK, Parmar P, Mullany LC, Lian SB, lacopino V, et al. Health and human rights in Chin State, Western Burma: a populationbased assessment using multistaged household cluster sampling. PLoS Med. 2011. doi:10.1371/journal.pmed.1001007.

12. ICF International. Demographic and Health Survey sampling and household listing manual. 2012. https://dhsprogram.com/pubs/pdf/DHSM4/ DHS6_Sampling_Manual_Sept2012_DHSM4.pdf. Accessed 10 Mar 2017.

13. Thomson DR, Ruktanonchai NW, Stevens FR, Castro M, Tatem AJ. GridSample: tools for grid-based survey sampling design. R package version 0.1.2. 2017. https://cran.r-project.org/package=gridsample. Accessed 10 Mar 2017.

14. United Nations Children's Fund (UNICEF). Designing and selecting the sample. In: Multiple indicator cluster surveys round 4. 2012. http://mics. unicef.org/tools?round=mics4. Accessed 10 Mar 2017.

15. United Nations (UN). Designing household survey samples: practical guidelines. Studies in methods series F No. 98. 2005. https://unstats. un.org/unsd/demographic/sources/surveys/Handbook23June05.pdf. Accessed 10 Mar 2017.

16. ICF International. Demographic and Health Survey sampling and household listing manual. 2012. https://dhsprogram.com/pubs/pdf/ DHSM4/DHS6_Sampling_Manual_Sept2012_DHSM4.pdf. Accessed 10 Mar 2017.

17. Grosh ME, Munoz J. A manual for planning and implementing the Living Standards Measurement Study Survey. LSMS Working Paper No. 126. 1996. http://documents.worldbank.org/curated/ en/363321467990016291/pdf/multi-page.pdf. Accessed 10 Mar 2017.

18. ICF International. Survey organization manual for Demographic and Health Surveys. 2012. http://dhsprogram.com/pubs/pdf/DHSM10/ DHS6_Survey_Org_Manual_7Dec2012_DHSM10.pdf. Accessed 13 May 2017.

19. Shannon HS, Hutson R, Kolbe A, Stringer B, Haines T. Choosing a survey sample when data on the population are limited: a method using Global Positioning Systems and aerial and satellite photographs. Emerg Themes Epidemiol. 2012. doi:10.1186/1742-7622-9-5.

20. Kamanga A, Renn S, Pollard D, Bridges DJ, Chirwa B, Pinchoff J, et al. Open-source satellite enumeration to map households: planning and targeting indoor residual spraying for malaria. Malar J. 2015. doi:10.1186/ s12936-015-0831-z.

21. Lohr SL. Sampling: design and analysis. 2nd ed. Boston: Brooks/Cole; 2009.

22. Census dates for all countries. 2020 World Population and Housing Census Programme, United Nations Statistics Division, Geneva. 2016. https:// unstats.un.org/unsd/demographic/sources/census/censusdates.htm. Accessed 10 Mar 2017.

23. Carr-Hill R. Missing millions and measuring development progress. World Dev. 2013. doi:10.1016/j.worlddev.2012.12.017.

24. GADM. Known problems. In: Global administrative areas v.2.8. 2015 http://www.gadm.org/problems. Accessed 3 Mar 2017.

25. Doxsey-Whitfield E, MacManus K, Adamo SB, Pistolesi L, Squires J, Borkovska $\mathrm{O}$, et al. Taking advantage of the improved availability of census data: a first look at the Gridded Population of the World, Version 4. Pap Appl Geogr. 2015. doi:10.1080/23754931.2015.1014272. 
26. Environmental Data Explorer: Gridded Population of the World. United Nations Environment Programme, Nairobi. 2006. http://geodata.grid. unep.ch/. Accessed 10 Mar 2017.

27. Balk D, Brickman M, Anderson B, Pozzi F, Yetman Y. Mapping global urban and rural population distributions: estimates of future global population distribution to 2015. 2005. http://www.fao.org/docrep/009/a0310e/ a0310e00.htm. Accessed 10 Mar 2017.

28. Pesaresi M, Ehrlich D, Florczyk AJ, Freire S, Julea A, Kemper T, et al. Operating procedure for the production of the Global Human Settlement Layer from Landsat data of the epochs 1975, 1990, 2000, and 2014. 2016. http:// publications.jrc.ec.europa.eu/repository/handle/JRC97705. Accessed 10 Mar 2017.

29. Facebook Connectivity Lab and Center for International Earth Science Information Network —CIESEN_Columbia University. High Resolution Settlement Layer (HRSL) [Internet]. Source imagery for HRSL 2016 DigitalGlobe. 2016. https://ciesin.columbia.edu/data/hrsl/. Accessed 10 Mar 2017.

30. Stevens FR, Gaughan AE, Linard C, Tatem AJ. Disaggregating census data for population mapping using random forests with remotely-sensed and ancillary data. PLoS ONE. 2015. doi:10.1371/journal.pone.0107042.

31. Dobson JE, Bright EA, Coleman PR, Durfee RC, Worley BA. LandScan: a global population database for estimating populations at risk. Photogramm Eng Remote Sens. 2000;66(7):849-57.

32. Azar D, Engstrom R, Graesser J, Comenetz J. Generation of fine-scale population layers using multi-resolution satellite imagery and geospatial data. Remote Sens Environ. 2013. doi:10.1016/j.rse.2012.11.022.

33. WorldPop Data. WorldPop, University of Southampton, Southampton UK. 2017. http://www.worldpop.org.uk/data/data_sources. Accessed 10 Mar 2017.

34. Gridded Population of the World v4. Center for International Earth Science Information Network, Columbia University, New York. 2016. http://sedac.ciesin.columbia.edu/data/collection/gpw-v4/sets/browse. Accessed 10 Mar 2017.

35. GHS Population Grid. European Commission, Brussels. 2017. http://ghsl. jrc.ec.europa.eu/ghs_pop.php. Accessed 18 May 2017.

36. Gridded Rural Urban Mapping Project v1. Center for International Earth Science Information Network, Columbia University, New York. 2006. http://sedac.ciesin.columbia.edu/data/set/grump-v1-population-count/ data-download. Accessed 10 Mar 2017.

37. LandScan Data Availability. Oak Ridge National Laboratories, Oak Ridge, Tennessee. 2017. http://www.ornl.gov/sci/landscan/landscan_data_avail. shtml. Accessed 02 Feb 2017.

38. Tatem AJ, Noor AM, Hay SI. Assessing the accuracy of satellite derived global and national urban maps in Kenya. Remote Sens Environ. 2005. doi:10.1016/.j.re.2005.02.001.

39. Linard C, Alegana V, Noor AM, Snow RW, Tatem AJ. A high resolution spatial population database of Somalia for disease risk mapping. Int J Health Geogr. 2010. doi:10.1186/1476-072X-9-45.

40. Tatem AJ. Mapping the denominator: spatial demography in the measurement of progress. Int Health. 2014. doi:10.1093/inthealth/ihu057.

41. Lu X, Wrathall DJ, Sundsøy PR, Nadiruzzaman M, Wetter E, lqbal A, et al. Detecting climate adaptation with mobile network data in Bangladesh: anomalies in communication, mobility and consumption patterns during cyclone Mahasen. Clim Change. 2016. doi:10.1007/s10584-016-1753-7.

42. Wilson R, Zu Erbach-Schoenberg E, Albert M, Power D, Tudge S, Gonzalez M, et al. Rapid and near real time assessments of population displacement using mobile phone data following disasters: the 2015 Nepal earthquake. PLoS Curr. 2015. doi:10.1371/currents.dis. d073fbece328e4c39087bc086d694b5c.

43. Deville P, Linard C, Martin S, Gilbert M, Stevens FR, Gaughan AE, et al. Dynamic population mapping using mobile phone data. Proc Natl Acad Sci. 2014. doi:10.1073/pnas. 1408439111.

44. Gething P, Tatem A, Bird T, Burgert-Brucker CR. Creating spatial interpolation surfaces with DHS data. DHS Spatial Analysis Reports 11. 2015. http:// dhsprogram.com/pubs/pdf/SAR11/SAR11.pdf. Accessed 10 Mar 2017.

45. Global Urban Footprint. DLR Earth Observation Center, Weßling. 2017. http://www.dlr.de/eoc/en/desktopdefault.aspx/tabid-11725/20508_read47944/. Accessed 10 Mar 2017.

46. Global Human Settlement City Model (GHS-SMOD). European Commission, Brussels. 2017. http://ghsl.jrc.ec.europa.eu/faq.php. Accessed 10 Mar 2017.
47. Schneider A, Friedl MA, Potere D. Mapping global urban areas using MODIS 500-m data: new methods and datasets based on "urban ecoregions". Remote Sens Environ. 2010. doi:10.1016/j.rse.2010.03.003.

48. Climate Change Institute Download Data. European Space Agency, Paris. 2016. http://maps.elie.ucl.ac.be/CCl/viewer/. Accessed 10 Mar 2017.

49. McIntyre NE, Knowles-Yánez K, Hope D. Urban ecology as an interdisciplinary field: differences in the use of "urban" between the social and natural sciences. In: Marzluff JM, Shulenberger E, Endlicher W, Alberti M, Bradley G, Ryan C, et al., editors. Urban ecology: an international perspective on the interaction between humans and nature. Boston: Springer; 2008.

50. Map Library. Map Maker Ltd, Campbeltown. 2007. http://www.maplibrary.org/library/stacks/Africa/index.htm. Accessed 10 Mar 2017.

51. DIVA-GIS. Hijmans R, Davis. 2016. http://www.diva-gis.org/gdata. Accessed 10 Mar 2017.

52. Tatem AJ, Campbell J, Guerra-Arias M, de Bernis L, Moran A, Matthews Z. Mapping for maternal and newborn health: the distributions of women of childbearing age, pregnancies and births. Int J Health Geogr. 2014. doi:10.1186/1476-072X-13-2.

53. Alegana VA, Atkinson PM, Pezzulo C, Sorichetta A, Weiss D, Bird T, et al. Fine resolution mapping of population age-structures for health and development applications. J R Soc Interface. 2015. doi:10.1098/ rsif.2015.0073.

54. Steele JE, Sundsøy RP, Pezzulo C, Alegana VA, Bird TJ, Blumenstock J, et al. Mapping poverty using mobile phone and satellite data. R Soc Interface. 2017. doi:10.1098/rsif.2016.0690

55. Ruktanonchai CW, Ruktanonchai NW, Nove A, Lopes S, Pezzulo C, Bosco $C$, et al. Equality in maternal and newborn health: modelling geographic disparities in utilisation of care in five East African countries. PLoS ONE. 2016. doi:10.1371/journal.pone.0162006.

56. National Institute of Statistics of Rwanda (NISR), Ministry of Health (MOH), ICF International. Rwanda Demographic and Health Survey 2010. 2012. http://www.measuredhs.com/pubs/pdf/FR259/FR259.pdf. Accessed 10 Mar 2017.

57. WorldPop. Rwanda population map metadata report. 2013. http://www. worldpop.org.uk/data/WorldPop_data/AllContinents/RWA-POP_metadata.html. Accessed 10 Mar 2017.

58. National Institute of Statistics of Rwanda (NISR). Fourth population and housing census. Thematic Report: population size, structure and distribution. 2012. http://statistics.gov.rw/old/publications/rphc4-thematicreport-population-size-structure-and-distribution. Accessed 10 Mar 2017.

59. Thomson DR. GridSample output: 2010 Rwanda DHS. Harvard Dataverse. 2017. doi:10.7910/DVN/MSCJOD.

60. Openshaw S. The modifiable areal unit problem. Norwick: Geo Books; 1983

61. Bosco C, Alegana V, Bird T, Pezzulo C, Bengtsson L, Sorichetta A, Steele J, Hornby G, Ruktanonchai C, Ruktanonchai N, Wetter E, Tatem AJ. Exploring the high-resolution mapping of gender-disaggregated development indicators. J R Soc Interface. 2017. doi:10.1098/rsif.2016.0825.

62. Lagonigro R, Oller R, Martori JC. A quadtree approach based on European geographic grids: reconciling data privacy and accuracy. SORT. 2017:41(1):139-58

63. Thompson SK. Adaptive cluster sampling. J Am Stat Assoc. 1990:85(412):1050-9.

64. Working Group for Mortality Estimation in Emergencies. Wanted: studies on mortality estimation methods for humanitarian emergencies, suggestions for future research. Emerg Themes Epidemiol. 2007. doi:10.1186/1742-7622-4-9.

65. Cutts FT, Claquin P, Danovaro-Holliday MC, Rhoda DA. Monitoring vaccination coverage: defining the role of surveys. Vaccine. 2016. doi:10.1016/j. vaccine.2016.06.053.

66. Luman ET, Worku A, Berhane Y, Martin R, Cairns L. Comparison of two survey methodologies to assess vaccination coverage. Int J Epidemiol. 2007. doi:10.1093/ije/dym025.

67. Siri JG, Lindblade KA, Rosen DH, Onyango B, Vulule JM, Slutsker L, et al. A census-weighted, spatially-stratified household sampling strategy for urban malaria epidemiology. Malar J. 2008. doi:10.1 186/1475-2875-7-39.

68. Wampler PJ, Rediske RR, Molla AR. Using ArcMap, Google Earth, and Global Positioning Systems to select and locate random households. Int J Health Geogr. 2013. doi:10.1186/1476-072X-12-3. 
69. Lin Y, Kuwayama DP. Using satellite imagery and GPS technology to create random sampling frames in high risk environments. Int J Surg. 2016. doi:10.1016/j.ijsu.2016.06.044.

70. Pearson AL, Rzotkiewicz A, Zwickle A. Using remote, spatial techniques to select a random household sample in a dispersed, semi-nomadic pastoral community: utility for a longitudinal health and demographic surveillance system. Int J Health Geogr. 2015. doi:10.1186/s12942-015-0026-4.

71. Escamilla V, Emch M, Dandalo L, Miller WC, Martinson F, Hofman I. Sampling at community level by using satellite imagery and geographical analysis. Bull World Health Organ. 2014. doi:10.2471/BLT.14.140756.

72. Tatem AJ, Noor AM, Hay SI. Defining approaches to settlement mapping for public health management in Kenya using medium spatial resolution satellite imagery. Remote Sens Environ. 2004. doi:10.1016/j. rse.2004.06.014

73. Tatem AJ, Campiz N, Gething PW, Snow RW, Linard C. The effects of spatial population dataset choice on estimates of population at risk of disease. Popul Heal Metr. 2011. doi:10.1186/1478-7954-9-4.
74. Hay SI, Noor M, Nelson A, Tatem J. The accuracy of human population maps for public health application. Trop Med Int Health. 2005. doi:10.1111/j.1365-3156.2005.01487.x.

75. Sorichetta A, Hornby GM, Stevens FR, Gaughan AE, Linard C, Tatem AJ. High-resolution gridded population datasets for Latin America and the Caribbean in 2010, 2015, and 2020. Sci Data. 2010. doi:10.1038/ sdata.2015.45.

76. Gaughan AE, Stevens FR, Linard C, Jia P, Tatem AJ. High resolution population distribution maps for Southeast Asia in 2010 and 2015. PLoS ONE. 2010. doi:10.1371/journal.pone.0055882.

77. Burgert CR, Colston J, Roy T, Zachary B. Geographic displacement procedure and georeferenced data release policy for the demographic and health surveys. 2013. https://dhsprogram.com/pubs/pdf/SAR7/SAR7.pdf. Accessed 10 Mar 2017

\section{Submit your next manuscript to BioMed Central and we will help you at every step:}

- We accept pre-submission inquiries

- Our selector tool helps you to find the most relevant journal

- We provide round the clock customer support

- Convenient online submission

- Thorough peer review

- Inclusion in PubMed and all major indexing services

- Maximum visibility for your research

Submit your manuscript at www.biomedcentral.com/submit 